\title{
Bone mineral density in elite rowers
}

\author{
Bronwen Lundy ${ }^{1 *}$, Larissa Trease ${ }^{2}$, Drew K. Michael ${ }^{1,3,4}$ \\ From World's Leading Rowing Sport Science and Medicine Conference - "Improving Performance Naturally" \\ Marlow, UK. 22-25 January 2015
}

\section{Background}

Bone mineral density (BMD) is known to be dependent on the loading pattern associated with a particular sport. High impact sports increases BMD at loaded sites with low impact sports having largely neutral findings [1]. The influence of high level rowing training has not been well explored with relatively small samples within a single category or discipline [2-5].

\section{Methods}

Subjects $(\mathrm{n}=125)$ were internationally competitive. Between 2011-2014 BMD was taken at the lumbar spine (L1-L4) and left femur, by Dual-energy X-ray absorptiometry (DXA, Lunar Prodigy, GE Healthcare), using the same scanner, and a qualified technician.
Ethics was approved by the Australian Institute of Sport Human Ethics committee. Subjects gave prior written informed consent. Descriptive statistics are reported as mean \pm standard deviation (range), Z-score and $\mathrm{T}$-score. Statistical analysis was performed using independent samples t-test, significance set at $\mathrm{p}<0.05$.

\section{Results}

A summary of findings is shown in Table 1. Overall, $5.6 \%$ of rowers had $Z \leq-1$ at the spine and $1.6 \%$ at the femur with none $Z<-2$. Both spine and femur BMD, $T$ and $\mathrm{Z}$ scores were lower for female lightweights than heavyweights. Male spine BMD and T score and femur $\mathrm{T}$ score was lower for lightweights relative to heavyweights.

Table 1. BMD in males and female rowers by weight category. Data are expressed mean \pm standard deviation (range)

\begin{tabular}{|c|c|c|c|c|c|c|}
\hline & Males & & & Females & & \\
\hline & Overall & Lightweight & Heavyweight & Overall & Lightweight & Heavyweight \\
\hline$n$ & 72 & 31 & 41 & 53 & 20 & 33 \\
\hline \multicolumn{7}{|l|}{ Spine } \\
\hline \multirow[t]{2}{*}{$\mathrm{g} / \mathrm{cm}^{2}$} & $1.33 \pm 0.13$ & $1.27 \pm 0.10 *$ & $1.38 \pm 0.12$ & $1.29 \pm 0.14$ & $1.19 \pm 0.09 *$ & $1.35 \pm 0.14$ \\
\hline & $(1.07-1.67)$ & $(1.07-1.46)$ & $(1.09-1.67)$ & $(1.05-1.67)$ & $(1.05-1.33)$ & $(1.07-1.67)$ \\
\hline \multirow[t]{2}{*}{ T score } & $0.9 \pm 1.1$ & $0.2 \pm 0.9 *$ & $1.3 \pm 1.0$ & $0.6 \pm 1.1$ & $-0.3 \pm 0.7 *$ & $1.0 \pm 1.1$ \\
\hline & $(-1.3-3.5)$ & $(-1.3-1.7)$ & $(-1.1-3.5)$ & $(-1.2-3.5)$ & $(-1.2-0.9)$ & $(-1.0-3.5)$ \\
\hline \multirow[t]{2}{*}{ Z score } & $0.7 \pm 1.0$ & $0.5 \pm 1.9$ & $0.8 \pm 1.0$ & $0.4 \pm 1.0$ & $0.1 \pm 0.7 *$ & $0.7 \pm 1.0$ \\
\hline & $(-1.5-3.2)$ & $(-1.2-1.9)$ & $(-1.5-3.2)$ & $(-1.3-3.3)$ & $(-1.0-1.2)$ & $(-1.3-3.3)$ \\
\hline \multicolumn{7}{|l|}{ Femur } \\
\hline \multirow[t]{2}{*}{$\mathrm{g} / \mathrm{cm}^{2}$} & $1.19 \pm 0.13$ & $1.16 \pm 0.13$ & $1.21 \pm 0.12$ & $1.12 \pm 0.13$ & $1.05 \pm 0.09 *$ & $1.17 \pm 0.13$ \\
\hline & $(0.97-1.58)$ & $(1.00-1.54)$ & $(1.02-1.58)$ & $(0.87-1.61)$ & $(0.87-1.23)$ & $(0.96-1.61)$ \\
\hline \multirow[t]{2}{*}{ T score } & $0.7 \pm 1.0$ & $0.4 \pm 0.9$ & $1.0 \pm 0.9$ & $0.5 \pm 0.9$ & $0.0 \pm 0.7 *$ & $0.8 \pm 0.8$ \\
\hline & $(-1-3.8)$ & $(-1.0-2.2)^{*}$ & $(-0.6-3.8)$ & $(-1.4-3.2)$ & $(-1.4-1.3)$ & $(-0.7-3.2)$ \\
\hline \multirow[t]{2}{*}{ Z score } & $0.5 \pm 0.9$ & $0.4 \pm 0.9$ & $0.5 \pm 1.0$ & $0.3 \pm 0.78$ & $0.2 \pm 0.8 *$ & $0.4 \pm 0.8$ \\
\hline & $(-1-3.4)$ & $(-0.9-2.3)$ & $(-1.0-3.4)$ & $(-1.1-3.0)$ & $(-1.1-1.6)$ & $(-0.8-3.0)$ \\
\hline
\end{tabular}

* significantly lower than for heavyweights within the same gender $(p<0.05)$

\footnotetext{
* Correspondence: bronwen.lundy@ausport.gov.au

${ }^{1}$ Australian Institute of Sport, Canberra, Australia

Full list of author information is available at the end of the article
} 


\section{Conclusion}

BMD of elite rowers appears to fall largely within the optimal range for the general population however lightweight rowers, tended to have lower BMD than their heavyweight counterparts at all measured sites at the spine and for females also at the femur.

\section{Authors' details}

${ }^{1}$ Australian Institute of Sport, Canberra, Australia. ${ }^{2}$ Principal Medical Officer, Rowing Australia, Canberra, Australia. ${ }^{3}$ Department of Physiotherapy, Faculty of Health, University of Canberra, Australia. ${ }^{4}$ Australian Centre for Research into Injury in Sport and its Prevention (ACRISP), Federation University, Australia.

Published: 11 August 2015

\section{References}

1. Scofield KL, Hecht S: Bone health in endurance athletes: runners, cyclists, and swimmers. Curr Sports Med Rep 2012, 11(6):328-334.

2. Dimitriou L, Weiler R, Lloyd-Smith R, Turner A, Heath L, James N, Reid A: Bone mineral density, rib pain and other features of the female athlete triad in elite lightweight rowers. BMJ Open 2014, 4(2):e004369.

3. Young KC, Kendall KL, Patterson KM, Pandya PD, Fairman CM, Smith SW: Rowing Performance, Body Composition, and Bone Mineral Density Outcomes in Collegiate-Level Rowers Following a Season of Concurrent Training. Int I Sports Physiol Perform 2014, 9(6):966-972.

4. Vinther A, Kanstrup IL, Christiansen E, Ekdahl C, Aagaard P: Testosterone and BMD in elite male lightweight rowers. Int I Sports Med 2008, 29(10):803-807.

5. Vinther A, Kanstrup IL, Christiansen E, Alkjaer T, Larsson B, Magnusson SP, Aagaard P: Exercise-induced rib stress fractures: influence of reduced bone mineral density. Scand J Med Sci Sports 2005, 15(2):95-99.

\section{Submit your next manuscript to BioMed Central} and take full advantage of:

- Convenient online submission

- Thorough peer review

- No space constraints or color figure charges

- Immediate publication on acceptance

- Inclusion in PubMed, CAS, Scopus and Google Scholar

- Research which is freely available for redistribution

Submit your manuscript at www.biomedcentral.com/submit 